\title{
Fabrication and stress relief modelling of GaN based MEMS test structures grown by $\mathrm{MBE}$ on $\mathrm{Si}(111)$
}

\author{
E. Sillero , D. López-Romero, A. Bengoechea, M. A. Sánchez-García, and F. Calle
}

Instituto de Sistemas Optoelectrónicos y Microtecnología and Dpto. Ing. Electrónica, E.T.S.I. Telecomunicación, UPM. 28040, Madrid, Spain

\begin{abstract}
The fabrication of III-N MEMS test structures, such as cantilevers, beams and stress-pointers, and the modelling of their deformation due to residual stress relief, is presented. GaN and $\mathrm{AlGaN} / \mathrm{GaN}$ structures were fabricated, either with one end and both ends clamped to the Si substrate (asymmetrical and symmetrical mechanical boundary conditions, respectively). The residual stress in the III-N layer was measured by photoluminescence and X-ray diffraction, and the stress relief induced deformation was analysed by a finite element method model. The deformations of the MEMS structures were used to calculate both the residual strains and the Young's
\end{abstract}

1 Introduction The properties of III-nitrides, such as wide bandgap, piezoelectric fields, high saturation velocity, thermal and chemical stability, make them very promising materials for MEMS applications, especially for devices operating at harsh environments. However, the lack of native substrates and the difficulty of selective etching of different III-N layers hinder the development of such devices. Growth of III-N layers on Si (111) usually accumulates large residual strains, due to the $17 \%$ lattice mismatch. The relaxation of large biaxial strains after the micromachining step, either tensile or compressive, leads to a deformation of the free standing structure which may render the device useless. These deformations are analysed in this work for several MEMS topologies.

2 Experimental Samples of $\mathrm{GaN} / \mathrm{Si}(111)$ and $\mathrm{Al}-$ $\mathrm{GaN} / \mathrm{GaN} / \mathrm{Si}(111)$ were grown by MBE. Prior to the growth of $\mathrm{GaN}$, a $30 \mathrm{~nm}$ thick high temperature $\left(800^{\circ} \mathrm{C}\right)$ AlN buffer was grown on top of the Si substrate in order to improve the nucleation and, therefore, the quality of the modulus of the material. One-end-clamped structures suffer from large deformations due to the uneven stress relaxation. During micromachining, the relaxation induces large upward buckling, as measured for fabricated devices and fitted by the FEM model. Two-end-clamped structures were also studied using different topologies and under-etching lengths of the clamped region. It is concluded that the deformation of such structures may be reduced with symmetrical mechanical boundary conditions and a small under-etched clamping region compared to the device dimensions.

$\mathrm{GaN}$ layer. The III-N layers were grown with thicknesses between $500 \mathrm{~nm}$ and $1 \mu \mathrm{m}$ for the GaN bulk layer and of approximately $20 \mathrm{~nm}$ for the AlGaN barrier. The Al molar fraction in the barrier was varied between $20 \%$ and $25 \%$, this variation was intended to analyse whether the barrier can withstand the etching process or not.

Various test structures were fabricated, including arrays of both end fixed beams, cantilevers and stress evaluation structures Among the stress evaluation structures symmetric $\mathrm{H}$-shaped beams and asymmetric pointers were chosen for this study, see Fig. 1. The central section of the Hshaped beam was varied in width in order to evaluate the deformation for different sections of relaxed material. The stress-pointers were used to asses the residual strain in the layer and together with the data of the $\mathrm{H}$-shaped beam calculate the residual stress and the Young's modulus of the material.

The MEMS were patterned using reactive ion etching with $\mathrm{SiCl}_{4}: \mathrm{Ar}_{2} \mathrm{SF}_{6}$ plasma released in HNA $\left(\mathrm{HF}: \mathrm{NOH}_{3}: \mathrm{CH}_{3} \mathrm{COOH}[2: 3: 8]\right.$ ) solutions and rinsed in 
uneven relaxation of the strain along the out of plane direction induces a bending momentum, responsible of the buckling. However, the simulation results yielded to smaller displacements than those observed, this deviation is currently under investigation.

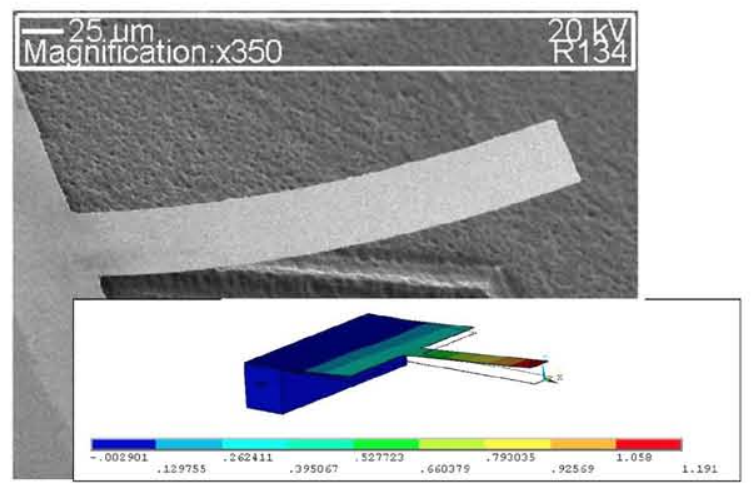

Figure $4 \mathrm{GaN}$ cantilever showing a large buckling due to uneven stress relaxation and the fitted FEM model (inset).

On the other hand, for the both end fixed beams and $\mathrm{H}$ shaped beams the observed deformations were significantly lower. For a beam with $100 \mu \mathrm{m}$ length and $10 \mu \mathrm{m}$ width the maximum out of plane deformation was measured to be less than $1 \mu \mathrm{m}$. This deformation is induced by the under-etching of the clamped regions of the beam. Simulations showed that if the under-etching is reduced, the buckling of the beam disappears. This has been confirmed by fabricating beams with different sizes but constant length-to-width ratio and under-etched length.
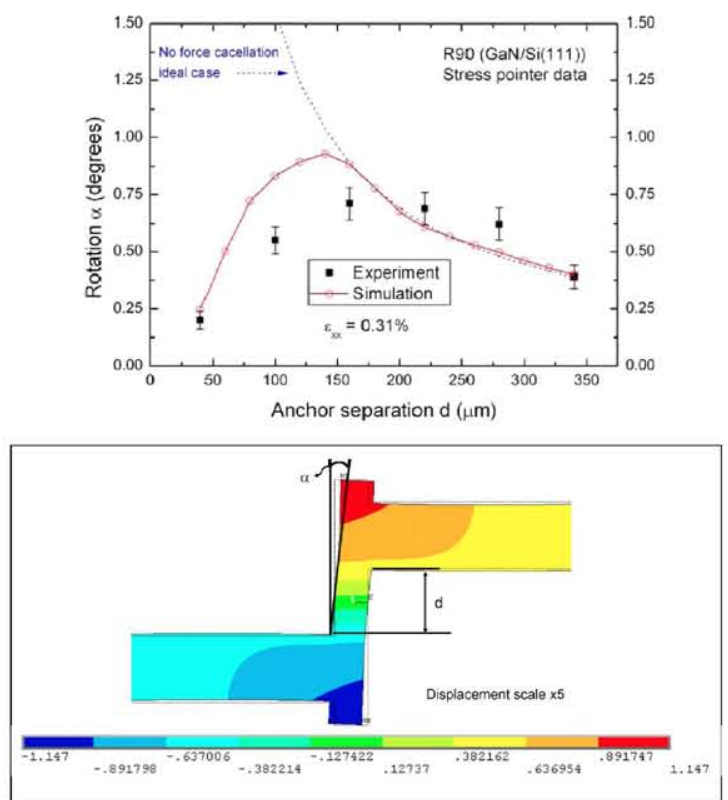

Figure 5 Experimental and calculated rotation of the stresspointers structures (above) and fitted FEM model (below). The dotted line in the figure shows the rotation if the material had no mechanical reaction to the deformation (ideal case).
The Young's modulus (E) and residual stress was determined from the observations made on the structures. The dotted line in Fig. 5 is used to extract the residual strain and the deviation due to the material reaction is used to extract the Young's modulus. The residual strain was estimated to be close to $0.3 \%$ in good agreement with the calculated from the $\mathrm{X}$ ray data, which was close to $0.27 \%$. The calculated Young's modulus had a significant dispersion between samples, but with high values for all the cases, in the range between 220 and $300 \mathrm{GPa}$. It is believed that the dispersion observed in the mechanical properties is due to the difficulty of growth of III-N layers on $\mathrm{Si}(111)$ without stress accommodation layers. Thus, the large number of defects present in the material are likely responsible of the lowering and subsequent dispersion of $\mathrm{E}$.

Finally, from the FEM model it was shown that the central section of the $\mathrm{H}$-shaped beams is completely relaxed. This allows the fabrication of piezoelectric inertial devices with high sensibility for both upward and downward displacements.

4 Conclusions It has been proved that it is possible to fabricate III-N MEMS structures on $\mathrm{Si}(111)$ with very good selectivity to the $(\mathrm{Al}, \mathrm{Ga}) \mathrm{N}$ layer using standard processing technology. Low deformation of the fabricated structures is expected if symmetrical mechanical boundary conditions are provided, and the under-etching of the clamping region is small compared to the device dimensions. The large residual stress is the main issue in this technology and must be reduced by means of the inclusion of stress accommodation layers during growth. Finally, it has been proven that nitrides may be used for stiff MEMS devices due to their high Young's modulus (around 280 GPa for the samples used in this study).

Acknowledgements This work was supported by Spanish projects Pr. TEC2004-05698-C02-01/MIC.MEC and FIT330100-2006-184. ES want to thank the FPU grant of the Spanish Ministry for Education and Science. We also want to thank E. J. Cho from TU Darmstadt for her help with the simulation work.

\section{References}

E. Zaus, M. Hermann, M. Stutzmann, and M. Eickhoff, phys. stat. sol. (RRL) 1, R10 (2007).

E. Sillero, D. Lopez-Romero, F. Calle, M. Eickhoff, J. F. Carlin, N. Grandjean, and M. Ilegems, Microelectron. Eng. 84, 1152 (2007).

R. P. Strittmatter, R. A. Beach, and T. C. McGill, Appl. Phys. Lett. 78, 3226 (2001).

T. Zimmermann, M. Neuburger, P. Benkart, F. J. HernandezGuillen, C. Pietzka, M. Kunze, I. Daumiller, A. Dadgar, A. Krost, and E. Kohn, IEEE Electron Dev. Lett. 27, 309 (2006).

B. P. van Drieënhuizen, J. F. L. Goosen, P. J. French, and R. F. Wolffenbuttel, Sens. Actuators A 37/38, 756 (1993).

E. Sillero, F. Calle, and M. A. Sanchez-Garcia, J. Mater. Sci. Mater. Electron. 16, 409 (2005). 
methanol in order to minimise sticking. For all the studied materials the selectivity with silicon of the wet etching was very high, since no etching of the nitride layer was at all detected as shown in Fig. 2.

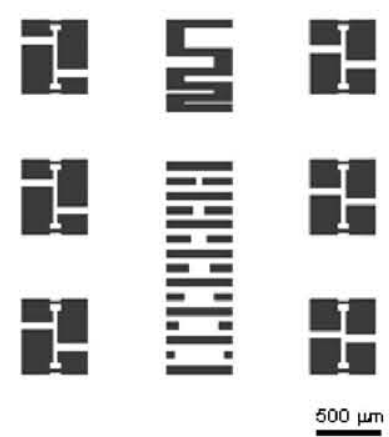

Figure 1 One of the masks used for the structural analysis. It may be observed the $\mathrm{H}$-shaped beams with varying central section, the six asymmetric stress-pointers with different distances between the anchor beams and an array of cantilevers with varying length to width aspect ratio.

A finite element method (FEM) model was developed using ANSYS 10.0 commercial software. The meshing was performed using 10 node tetrahedral elements with anisotropic elastic properties and pre-stress capabilities (SOLID187). The residual stress applied to the model was customized to include the stress relaxation along the growth direction. The AlGaN layer was not included in the simulation because the meshing of such a thin layer with compatible elements is very difficult and may lead to convergence problems in the calculation.

The samples were characterised using scanning electron microscopy (SEM), high resolution $\mathrm{X}$ ray diffraction (HRXRD) and photoluminescence (PL) in order to asses the residual strain in the III-N layer. The obtained residual stress values were used as input to the FEM model in order to fit the observed deformations of the structures and, therefore, study the relaxation mechanism of the micromachined devices.

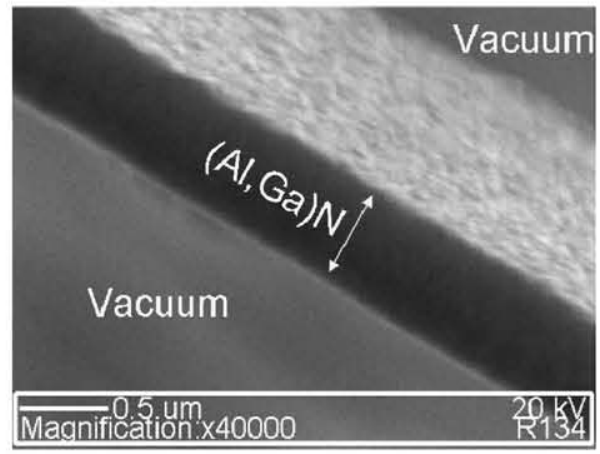

Figure 2 Cross section SEM image of a freestanding $\mathrm{AlGaN} / \mathrm{GaN}$ beam showing no apparent etching either at the $\mathrm{Ga}-$ face $\mathrm{AlGaN}$ or at the $\mathrm{N}$-face $\mathrm{GaN}$.

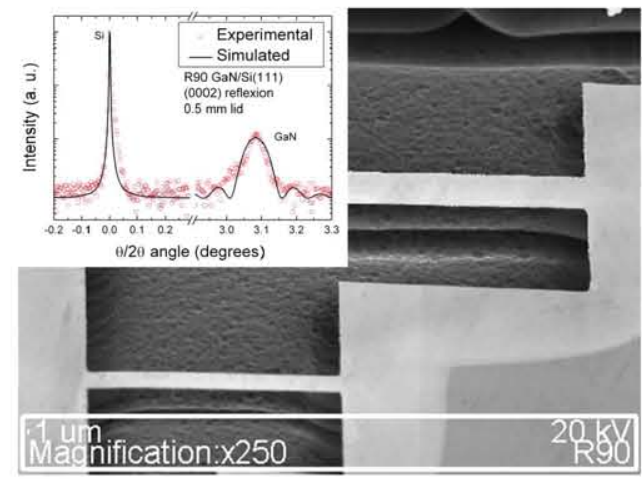

Figure $3 \mathrm{GaN}$ micromachined beams with different lengths. At the step between the beams the under-etched tub at the clamping area is clearly visible. This under-etching of the Si substrate defines the boundary of the mechanically free structure. At the inset an HRXRD diffractogram shows a small shift of the GaN reflection, revealing a certain amount of residual tensile strain.

3 Results and discussion All samples had tensile residual stress as derived from PL measurements (FXA energy at $10 \mathrm{~K}$ ranging from $3.4692 \mathrm{eV}$ to $3.4699 \mathrm{eV}$ ), the broadness of the peaks from the non optimised material hindered the accuracy of the strain calculation from this data. Therefore, HRXRD data was used to calculate the residual strain, which was about $0.27 \%$ (nearly $98.4 \%$ of the pseudomorphic strain was relaxed during growth leading to a lattice constant $\mathrm{a}=0.31962 \mathrm{~nm}$ ). From these observations the residual stress was calculated to be in the range between $750 \mathrm{MPa}$ and $960 \mathrm{MPa}$ for all the studied samples. This value is very high for the usual MEMS structures and is expected to significantly affect the performance of MEMS devices if growth is not optimised to include stress accommodation layers (such as AlN/GaN superlattices).

The isotropic wet etch step employed for the removal of the sacrificial layer induced an under-etching of the clamping regions of the devices, affecting their mechanical properties. The etched tub under the MEMS structure extended its limits further than those defined in the mask, see Fig. 3. Therefore a cornice of material is formed at the borders of the structure.

The micromachined cantilevers and one end clamped structures suffered from severe upward curling after release. The same effect is also observed in the asymmetric stress-pointers where the mechanical boundary conditions are not geometrically balanced to cancel each other. The relaxation degree of the layer along the $\mathrm{z}$ direction was calculated using the FEM model and the buckling was approximated (Fig. 4). The ISTRESS command was issued in order to generate a constant initial stress applied on the nodes of the model and no special restrains were set on the upper and lower area to allow a free relief of the residual strain. It was shown that the bending is due to a stress gradient arising from the complete relaxation of the upper surface of the GaN layer, whereas the lower surface remains stressed because it is clamped to the Si substrate. Thus, the 